\title{
The Endophytic Bacteria Bacillus velezensis Lle-9, Isolated from Lilium leucanthum, Harbors Antifungal Activity and Plant Growth-Promoting Effects
}

\author{
Mohammad Sayyar Khan ${ }^{1,2}$, Junlian Gao', Xuqing Chen ', Mingfang Zhang', Fengping Yang', \\ Yunpeng Du' ${ }^{1}$, The Su Moe ${ }^{1,3}$, Iqbal Munir ${ }^{2}$, Jing Xue ${ }^{1 *}$, and Xiuhai Zhang ${ }^{1 *}$ \\ 'Beijing Agro-Biotechnology Research Center, Beijing Academy of Agriculture and Forestry Sciences, Beijing \\ 100097, P.R. China \\ ${ }^{2}$ Genomics and Bioinformatics Division, Institute of Biotechnology and Genetic Engineering (IBGE), The University \\ of Agriculture, Peshawar 25000 Khyber Pakhtunkhwa, Pakistan \\ ${ }^{3}$ Pharmaceutical Research Laboratory, Biotechnology Research Department, Ministry of Education, Mandalay \\ Division, Kyaukse 05151, Myanmar
}

Bacillus velezensis is an important plant growth-promoting rhizobacterium with immense potential in agriculture development. In the present study, Bacillus velezensis Lle-9 was isolated from the bulbs of Lilium leucanthum. The isolated strain showed antifungal activities against plant pathogens like Botryosphaeria dothidea, Fusarium oxysporum, Botrytis cinerea and Fusarium fujikuroi. The highest percentage of growth inhibition i.e., $68.56 \pm 2.35 \%$ was observed against Fusarium oxysporum followed by $63.12 \pm \mathbf{2 . 8 3} \%, 61.67 \pm 3.39 \%$ and $\mathbf{5 5 . 8 2} \pm \mathbf{2 . 7 6} \%$ against Botrytis cinerea, Botryosphaeria dothidea, and Fusarium fujikuroi, respectively. The ethyl acetate fraction revealed a number of bioactive compounds and several were identified as antimicrobial agents such as diketopiperazines, cyclo-peptides, linear peptides, latrunculin A, 5a-hydroxy-6-ketocholesterol, (R)-S-lactoylglutathione, triamterene, rubiadin, moxifloxacin, 9-hydroxy-5Z,7E, 11Z,14Zeicosatetraenoic acid, D-erythro-C18-Sphingosine, citrinin, and 2arachidonoyllysophosphatidylcholine. The presence of these antimicrobial compounds in the bacterial culture might have contributed to the antifungal activities of the isolated $B$. velezensis Lle9. The strain showed plant growth-promoting traits such as production of organic acids, ACC deaminase, indole-3-acetic acid (IAA), siderophores, and nitrogen fixation and phosphate solubilization. IAA production was accelerated with application of exogenous tryptophan concentrations in the medium. Further, the lily plants upon inoculation with Lle-9 exhibited improved vegetative growth, more flowering shoots and longer roots than control plants under greenhouse condition. The isolated $B$. velezensis strain Lle- 9 possessed broad-spectrum antifungal activities and multiple plant growth-promoting traits and thus may play an important role in promoting sustainable agriculture. This strain could be developed and applied in field experiments in order to promote plant growth and control disease pathogens.

Keywords: Endophytic bacteria, antifungal activity, secondary metabolites, plant growth promotion

\section{Introduction}

The plant-associated bacteria termed as 'plant growth-promoting rhizobacteria (PGPRs)' have long been considered as important players in growth promotion and disease resistance in plants [1]. These PGPRs utilize several mechanisms to exert direct or indirect beneficial effects on associated plants. Synthesis of plant growth hormones, phosphate solubilization, nitrogen fixation, siderophores and ACC deaminase production are some of the key mechanisms through which these PGPRs promote plant growth $[2,3]$. The PGPR-mediated disease resistance is conferred through various mechanisms either by blocking the rhizopshere colonization of pathogens or through secretion of antimicrobial compounds that degrade the cell walls of pathogens $[4,5]$.

Among the isolated PGPRs, several species of the genus Bacillus have been characterized as producing a variety of compounds and metabolites with antimicrobial and plant growth-promoting effects [6,7]. For example, species such as Bacillus licheniformis, Bacillus amyloliquefaciens, and Bacillus subtilis have been proved very effective in 
controlling fungal diseases [8]. Some commercially available strains of these species are used as biofertilizers and biocontrol agents $[9,10]$. In this connection, Bacillus velezensis is an important member of the Bacillus genus, and was first described as a heterotypic synonym of B. amyloliquefaciens [11]. Later, several other Bacillus species were reclassified as Bacillus velezensis based on genomics and DNA hybridization matches [12]. Several strains of B. velezensis have been isolated and characterized for their plant growth promotion and production of antifungal metabolites $[13,9]$. Due to these properties, some strains of $B$. velezensis have been utilized in agriculture as plant growth promoters and biological control agents $[14,15,12]$.

Lily, belonging to family Liliaceae, has significant aesthetic, medicinal and edible value. The genus Lilium contains about 100 species and is native to Asia, Europe, and North America. China, with 55 species, is considered the world's center of diversity when it comes to wild Lilium [16]. Lily bulbs have a centuries-long history of extensive utilization as a food source and traditional medicine in China. They have been used to provide nourishment as well as health-promoting properties to treat bronchitis and pneumonia $[17,18]$. In addition, various Lilium species can also withstand harsh environmental conditions like drought and biotic stresses. In this connection, the role of plant-associated rhizosphere and endophytic microbes is crucial as some of the microbial metabolites seem to be characteristics of the biotype [19]. It was reported that some bioactive natural products recovered from plants had close similarities with products produced by the associated endophytes [20]. To the best of our knowledge, no reports are available of endophytic bacteria isolation from the bulbs of Lilium species up to date.

In the current study, an endophytic bacterial strain of Bacillus velezensis was isolated and identified from the bulbs of Lilium leucanthum. The isolated strain was further evaluated for antifungal, secondary metabolites and plant growth-promoting effects.

\section{Materials and Methods}

\section{Sample Sterilization and Endophyte Isolation}

Bulbs of Lilium leucanthum were collected from the experimental fields of Beijing Agro-Biotechnology Research Center, Academy of Agriculture and Forestry Sciences, China in December 2018.

The bulbs' preparation, sterilization and isolation of endophyte were carried out using a previously described method [21]. The bulbs were peeled off, and the inner portions were washed with tap water for 5 min followed by treatment with $70 \%(\mathrm{v} / \mathrm{v})$ ethanol for $1 \mathrm{~min}$. The samples were then immersed in $10 \%$ (concentration of active chlorine) $\mathrm{NaClO}$ solution for $20 \mathrm{~min}$ and washed with sterile distilled water three times. After surface sterilization, the bulb portions were cut aseptically into approximately $1 \mathrm{~cm} \times 1 \mathrm{~cm}$ pieces and placed on LB agar media plates. The plates were incubated at $30^{\circ} \mathrm{C} \pm 1^{\circ} \mathrm{C}$ for $2-3$ days until bacterial growth started on the cut bulb portions. The individual bacterial colonies appeared on bulb portions were aseptically inoculated into fresh LB broth and incubated at $30^{\circ} \mathrm{C} \pm 1^{\circ} \mathrm{C}$ until pure cultures were obtained by serial sub-culturing.

\section{Morphological and Molecular Identification of Endophytic Bacteria}

The isolated bacterial strain Lle-9 was characterized using colony morphology, growth pattern, Gram staining and scanning electron microscopic (SEM) analysis. The Gram reaction was performed as previously described [22]. Cell morphology of the isolate was determined using an SU8010 Field-Emission Scanning Electron Microscope (SEM, Hitachi, Japan).

For molecular analysis, the endophytic strain Lle- 9 was cultured in LB broth for $24 \mathrm{~h}$, incubated at $30^{\circ} \mathrm{C}$ in a shaker with $220 \mathrm{rpm}$. The culture was then centrifuged at 4,000 $\times g$ for $15 \mathrm{~min}$. Supernatant was discarded and the cell pellet was used for genomic DNA extraction using the Bacterial Genomic DNA Isolation Kit (SolarBio, China) in accordance with the manufacturer's protocols. The isolated endophyte was identified by the sequences of $16 \mathrm{~S}$ ribosomal RNA (rRNA) genes. A sequence of about 1,500 bp was amplified from genomic DNA using primers P027F and 1378R specific for the 16S ribosomal RNA genes. A $25 \mu \mathrm{l} \mathrm{PCR} \mathrm{reaction} \mathrm{contained} 1 \mu \mathrm{l}$ (0.5-10.0 ng) of template DNA, $0.2 \mu \mathrm{M}$ of each primer, P027F (5'-GAGAGTTTGATCCTGGCTAG-3) and 1378R (5'-CGGTGT GTACSSGGCCCGGGAACG-3'), $200 \mu \mathrm{M}$ of each dNTP, 10X buffer, $2 \mathrm{mM} \mathrm{MgSO}_{4}$, and $1 \mathrm{U}$ High-Fidelity KOD Taq DNA Polymerase. The cycle parameters were as follows: initial denaturation at $94^{\circ} \mathrm{C}$ for $4 \mathrm{~min} ; 30$ cycles of denaturation for $30 \mathrm{sec}$ at $94^{\circ} \mathrm{C}$, annealing for $1 \mathrm{~min}$ at $63^{\circ} \mathrm{C}$, and extension for $1 \mathrm{~min}$ at $68^{\circ} \mathrm{C}$; and a final overall extension for $7 \mathrm{~min}$ at $68^{\circ} \mathrm{C}$. The PCR product was purified using the QIAquick PCR Purification Kit (Qiagen, Germany) and was then sent to Beijing Biomed Gene Technology Co., Ltd. for sequencing. Sequences were BLAST searched against homologous bacterial 16S ribosomal RNA sequences using NCBI. The determined sequences were aligned using ClustalW, and phylogenetic trees were constructed based on Neighbor-Joining (NJ) and Maximum Likelihood (ML) algorithms using the MEGA 7 software [23]. The nucleotide sequence was then submitted to GenBank under accession number MN461530.1.

\section{Antifungal Activity}

In vitro antifungal assay was conducted to test the antagonistic effects of the isolated endophytic strain Lle-9 against four strains of pathogenic fungi, including Botryosphaeria dothidea, Fusarium oxysporum, Botrytis cinerea and Fusarium fujikuroi. The antifungal bioassays were conducted based on the dual culture method as previously described [24]. Zones of inhibition were calculated using the formula: $\%$ of growth inhibition $=[(\mathrm{C}-\mathrm{T}) / \mathrm{C}] \times 100$, where, $\mathrm{C}$ is the radial growth of the test pathogen in the control plates $(\mathrm{mm})$, and $\mathrm{T}$ is the radial growth of the test pathogen in the test plates $(\mathrm{mm})$. The experiment was repeated thrice. 


\section{Ethyl Acetate Extraction of Secondary Metabolites}

Secondary metabolites of the Lle-9 strain extracted by solvent partition method. The strain was grown in LB broth at $30^{\circ} \mathrm{C}$ and $150 \mathrm{rpm}$ shaking for 5-6 days. The broth cultures were then centrifuged at $10,000 \times g, 4^{\circ} \mathrm{C}$ for 10 $\mathrm{min}$. The supernatant was filtered through a $0.2 \mu \mathrm{m}$ syringe filter. An equal volume of the filtrate and ethyl acetate was taken into the separating funnel and shaken for the complete extraction. The solvent phase that contains secondary metabolites was separated from the aqueous phase and solvent was evaporated to dryness to yield the crude extracts. The crude extract, about $20 \mathrm{mg}$, was re-dissolved in $1 \mathrm{ml}$ of $70 \%$ methanol. Then, $500 \mu \mathrm{l}$ of dissolved extract was filtered through a $0.2 \mu \mathrm{m}$ syringe filter before ultra high-performance liquid chromatography LTQ XL linear ion trap mass spectrometry/mass spectrometry (UHPLC-LTQ-XL-IT-MS/MS) analysis.

\section{UHPLC-LTQ-XL-IT-MS/MS Analysis for Secondary Metabolite Profiling}

UHPLC-LTQ-IT-MS/MS analysis was performed using the method partially adapted from Lee et al. [25]. The Thermo Fischer Scientific LTQ XL linear ion trap mass spectrometry consisted of an electrospray interface (Thermo Fischer Scientific, USA) coupled with a DIONEX UltiMate 3000 RS Pump, RS Auto Sampler, and RS Column Compartment (Dionex Corporation, USA) that were used for secondary metabolite profiling of the fungal extracts. Sample was separated on a Thermo Scientific Hypersil GOLD C18 column with $1.9 \mu \mathrm{m}$ particle size. The mobile phase consisted of $\mathrm{A}(0.1 \%(v / v)$ formic acid in water $)$ and $\mathrm{B}(0.1 \%(v / v)$ formic acid in acetonitrile and the gradient conditions were increased from $10 \%$ to $100 \%$ of solvent B. Scanning was set to start after 1 min to source. Solvent gradient time was set over $19 \mathrm{~min}$, and re-equilibrated to the initial condition for 4 min by setting the divert valve to waste. The flow rate was set at $0.3 \mathrm{ml} / \mathrm{min}$ and the injection volume was $10 \mu \mathrm{l}$. Temperature of the column during measurement was maintained at $35^{\circ} \mathrm{C}$. Ion trap was performed in positive and full-scan ion modes within a range of $150-1,000 \mathrm{~m} / z$. The operating parameters were as follows: source voltage, $\pm 5 \mathrm{kV}$; capillary voltage, $39 \mathrm{~V}$; capillary temperature, $275^{\circ} \mathrm{C}$; auxiliary gas flow rate $10-20$ arbitrary units; sheath gas flow rate 40-50 arbitrary units; spray voltage $4.5 \mathrm{kV}$. Tandem MS (MS/MS) analysis was performed by scan-type turbo data-dependent scanning (DDS) under the same conditions used for MS scanning for the six most intense ions using the Nth order double play mode. MS data were acquired by Xcalibar software (Thermo Fischer Scientific, USA).

\section{Putative Identification of Secondary Metabolites}

Putative identification of secondary metabolites was done using molecular networking workflow from the GNPS website (https://gnps. ucsd.edu) [26]. Raw LC-MS files were converted into mzXML using ProteoWizard 3.0.19140 [27], and the mzXML file was uploaded to GNPS. A molecular network was created using the default parameters. The spectra in the network were then searched against GNPS' spectral libraries. The library spectra were filtered in the same manner as the input data. All matches kept between network spectra and library spectra were required to have a score above 0.7 and at least 6 matched peaks.

\section{Plant Growth-Promoting (PGP) Assays}

For plant growth-promoting assays, the bacterial strain Lle-9 was cultured in $1 \mathrm{ml} \mathrm{LB}$ media at $30^{\circ} \mathrm{C}$ for $48 \mathrm{~h}$ with $200 \mathrm{rpm}$ shaking. The culture was then centrifuged at $4,000 \times g$ for $10 \mathrm{~min}$ at room temperature. The supernatant was removed and the cell pellet was washed thrice with $1 \mathrm{ml} 10 \mathrm{mM} \mathrm{MgSO}_{4}$. The pellet was then resuspended in $650 \mu \mathrm{l}$ of $\mathrm{MgSO}_{4}$ and was used for PGP assays.

\section{ACC Deaminase Detection}

The isolated strain Lle-9 was assayed for the production of 1-aminocyclopropane-1-carboxylate (ACC) deaminase according to the method previously developed by Belimov et al. [28] with minor modifications [29]. The color change from yellow to brown was considered as positive.

\section{Organic Acid Production Assay}

Organic acids in the isolated strain Lle-9 were detected according to the protocol developed by Cunningham and Kuiack [30]. About $50 \mu \mathrm{l}$ of the bacterial suspension in $\mathrm{MgSO}_{4}(10 \mathrm{mM})$ was inoculated in $800 \mu \mathrm{l}$ of Sucrose Tryptone medium (ST) containing $20 \mathrm{~g} \mathrm{l}^{-1}$ sucrose and $5 \mathrm{~g} \mathrm{l}^{-1}$ tryptone. The ST medium was supplemented with $10 \mathrm{ml}$ of trace element solution. Samples were incubated at $30^{\circ} \mathrm{C}$ and $200 \mathrm{rpm}$ shaking for 5 days. After incubation, organic acids in samples were detected by adding $100 \mu \mathrm{l}$ of $0.1 \%$ alizarine red $\mathrm{S} \mathrm{pH}$ indicator. After $15 \mathrm{~min}$, samples with yellow color were considered as positive. Pink-colored samples indicated negative results.

\section{Indole Acetic Acid (IAA) Detection}

Indole acetic acid (IAA) production in the isolated strain Lle-9 was assayed according to the method of Gordon and Weber [31]. A bacterial suspension of $150 \mu$ l prepared in $\mathrm{MgSO}_{4}(10 \mathrm{mM})$ was inoculated in $3 \mathrm{ml}$ of $1 / 10$ diluted 869-rich medium. The medium was supplemented with various tryptophan concentrations of $0 \mathrm{mg} \mathrm{ml}^{-1}$, $2 \mathrm{mg} \mathrm{ml}^{-1}, 4 \mathrm{mg} \mathrm{ml}^{-1}$, and $6 \mathrm{mg} \mathrm{ml}^{-1}$. Samples were incubated at $30^{\circ} \mathrm{C}$ for 4 days with $150-180 \mathrm{rpm}$ shaking in the dark. After incubation, the cultures were centrifuged at 4,000 $\times \mathrm{g}$ for $20 \mathrm{~min}$. About $1 \mathrm{ml}$ of the supernatant was

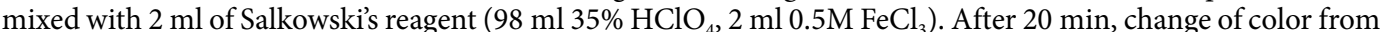
yellow to pink was considered as positive for IAA production. Quantitative measurement of indole acetic acid in the samples was conducted by measuring OD at $530 \mathrm{~nm}$ in a spectrophotometer. The IAA quantities in samples were measured based on a standard curve of known values (Fig. S1). 


\section{Siderophore Detection}

The siderophore production in the strain Lle-9 was evaluated through both qualitative and quantitative tests. The bacterial cells were cultured in liquid 284 medium with chrome azurol S (CAS) shuttle solution, according to the described method [32]. About $50 \mu \mathrm{l}$ of the bacterial suspension in $10 \mathrm{mM} \mathrm{MgSO}_{4}$ was added to $800 \mu \mathrm{l}$ of 284 medium prepared with three different iron concentrations. The iron concentrations used were: $0 \mu \mathrm{M}, 0.25 \mu \mathrm{M}$, and $3 \mu \mathrm{M} \mathrm{Fe}(\mathrm{III})$ citrate. Samples were incubated at $30^{\circ} \mathrm{C}$ for 5 days with $150 \mathrm{rpm}$ shaking. After incubation, $100 \mu \mathrm{l}$ of the blue Chromium Azurol S (CAS) reagent was added to samples followed by incubation for $4 \mathrm{~h}$ at room temperature. After incubation, the change of color from blue to orange/yellow was considered as positive. Siderophore concentrations in all samples were further measured at $630 \mathrm{~nm}$. The siderophore quantities were measured as \% of siderophore units by the formula: \% of siderophore units = $\mathrm{Ar}-\mathrm{As} / \mathrm{Ar} * 100$ where, "Ar" is the absorbance of reference (CAS reagent); and "As" is the absorbance of sample at $630 \mathrm{~nm}$. The ability of the strain to produce siderophores was further confirmed through qualitative test using CAS agar assay. All assays were carried out in triplicates.

\section{Nitrogen Fixation}

Nitrogen fixing ability of the isolated strain Lle-9 was evaluated on nitrogen-deficient malate medium (NFM). Escherichia coli strain DH5a was used as negative control. A single colony of each endophytic strain Lle-9 and E. coli strain DH5a grown on solid LB medium were streaked onto solid nitrogen-deficient malate medium. Composition of this medium was: $0.1 \mathrm{gl}^{-1} \mathrm{NaCl}, 0.02 \mathrm{gl}^{-1} \mathrm{CaCl}_{2}, 0.01 \mathrm{gl}^{-1} \mathrm{FeCl}_{3}, 0.4 \mathrm{gl}^{-1} \mathrm{KH}_{2} \mathrm{PO}_{4}, 0.5 \mathrm{gl}^{-1} \mathrm{~K}_{2} \mathrm{HPO}_{4}$, $0.2 \mathrm{~g} \mathrm{l}^{-1} \mathrm{MgSO}_{4} \cdot 7 \mathrm{H}_{2} \mathrm{O}, 0.002 \mathrm{~g} \mathrm{l}^{-1} \mathrm{Na}_{2} \mathrm{MoO}_{4} \cdot 2 \mathrm{H}_{2} \mathrm{O}, 5 \mathrm{~g} \mathrm{l}^{-1}$ sodium malate, $15 \mathrm{~g} \mathrm{l}^{-1}$ agar, $\mathrm{pH} 7.2-7.4$ using $\mathrm{KOH}$ ) supplemented with $50 \mathrm{mg} \mathrm{l}^{-1}$ yeast extract [33]. Once colonies of the isolated strain appeared on NFM media, a resulting single colony was re-streaked onto NFM to confirm the ability to fix nitrogen [34]. Plates were incubated for 7 days at $28^{\circ} \mathrm{C}$.

\section{Phosphate Solubilization}

Phosphate solubilization was evaluated according to the method developed by Mehta and Nautiyal [35]. The B. velezensis strain Lle- 9 was cultured on solid NBRIP medium $\left(10 \mathrm{~g} \mathrm{l}^{-1}\right.$ glucose, $5 \mathrm{~g} \mathrm{l}^{-1} \mathrm{Ca}_{3}\left(\mathrm{PO}_{4}\right)_{2}, 5 \mathrm{~g} \mathrm{l}^{-1} \mathrm{MgCl}_{2}$, $0.1 \mathrm{gl}^{-1}\left(\mathrm{NH}_{4}\right)_{2} \mathrm{SO}_{4}, 0.25 \mathrm{gl}^{-1} \mathrm{MgSO}_{4} \cdot 7 \mathrm{H}_{2} \mathrm{O}, 0.2 \mathrm{gl}^{-1} \mathrm{KCl}, 15 \mathrm{gl}^{-1}$ agar). The ability of the strain Lle- 9 to use inorganic phosphate in the form of $\mathrm{Ca}_{3}\left(\mathrm{PO}_{4}\right)_{2}$ as a sole phosphate was determined. Plates were incubated for 14 days at $28^{\circ} \mathrm{C}$.

\section{Experimental Design of Greenhouse Test}

The growth-promoting effects of the isolated endophytic bacterial strain Lle-9 were evaluated on the Asiatic lilium hybrids 'Tresor.' Same-sized bulbs with normal and healthy appearance were selected from the storage house at $4^{\circ} \mathrm{C}$. For inoculation, the isolated strain Lle- 9 was cultured in $5 \mathrm{ml} \mathrm{LB}$ for $10-15 \mathrm{~h}$ followed by further inoculation in $50 \mathrm{ml} \mathrm{LB}$ for $24 \mathrm{~h}$ at $30^{\circ} \mathrm{C}$ with $220 \mathrm{rpm}$ shaking. After incubation, the culture was re-inoculated in $400 \mathrm{ml} \mathrm{LB}$ and was kept to grow at $30^{\circ} \mathrm{C}$ for $24 \mathrm{~h}$. This culture was then diluted 10 times with normal water and bulbs of Tresor variety were soaked in the diluted culture for $40 \mathrm{~min}$. The non-inoculated bulbs, soaked in simple LB, were used as controls. Soil pots of sizes $20 \times 30 \mathrm{~cm}$ were prepared with soil mix of peat moss, perlite, and vermiculite in a ratio of 2:1:1. Three lily bulbs, either inoculated or non-inoculated control were sown in each soil pot. Pots were kept in a completely randomized design (CRD). Each treatment contained 5 pots. Pots were kept in plastic trays with holes in the bottom. The plastic trays were watered with equal amounts of normal tap water at regular intervals. Morphological data such as number of flowering shoots, plant height, leaf length, leaf width, bulb size and weight and root length were taken at the peak vegetative and reproductive stage.

\section{Statistical Analysis}

The data obtained were subjected to analysis of variance (ANOVA). Means were compared with Student's $t$-test at a probability of $\alpha=0.05$.
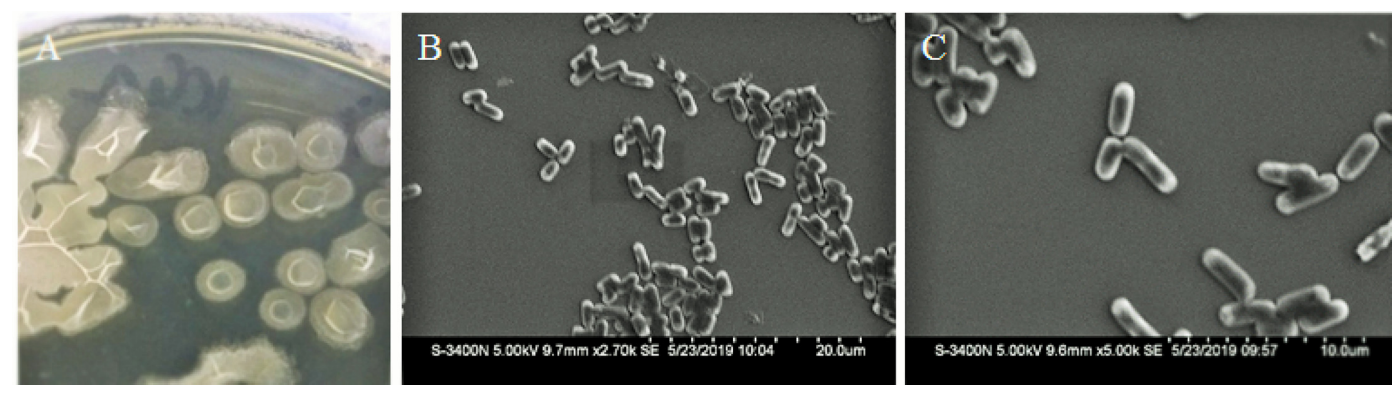

Fig. 1. Colony morphology and scanning electron microscope (SEM) analysis of the endophytic bacterial strain of B. velezensis isolated from L. leucanthum. Strain Lle-9 produced whitish, chalky-colored colonies on LB agar plates (A). The isolate is of small rod-shaped structures $(\mathbf{B}, \mathbf{C})$. 


\section{Results}

\section{Isolation and Identification of $B$. velezensis Strain Lle-9}

In our study, several bacterial endophytes were isolated from the bulb samples of Lilium leucanthum. Based on the antifungal assays, the isolated endophytic strain Lle- 9 of B. velezensis was selected for further assays. The isolated strain Lle-9 formed off whitish chalky colonies with internal whitish circular lines (Fig. 1A), as a grampositive and spore-forming bacterium and exhibited small rod-shaped structures typical of the genus Bacillus as revealed by Scanning Electron Microscopic (SEM) analysis (Figs. 1B and 1C).

Molecular analysis indicated that the isolated strain Lle-9 belongs to the genus Bacillus. The BLAST results revealed that the 16S rRNA gene sequence was closely related to Bacillus velezensis. Based on the maximum likelihhod phylogenetic tree constructed with the 16S rRNA similarity (\%), the Lle-9 strain revealed 99.34\% similarity with Bacillus velezensis strain CR-502 (T), (AY603658) (Fig. 2). The 16S rRNA gene sequence of the isolated strain shared high similarities with other Bacillus species such as $99.25 \%$ with Bacillus siamensis, $99.25 \%$ with Bacillus nakamurai, and 99.16\% with both Bacillus amyloliquefaciens and Bacillus subtillus. The 16S rRNA gene sequence of the isolated strain Lle-9 was submitted to GenBank under accession number MN461530.1.

\section{Antifungal Activity Analysis}

The isolated endophytic strain Lle-9 showed high potential of broad-spectrum antifungal activities against the tested phytopathogens, i.e., Botryosphaeria dothidea, Fusarium oxysporum, Fusarium fujikuroi, and Botrytis cinerea (Fig. 3A). These pathogenic strains were previously tested in an in vitro study for their pathogenicity

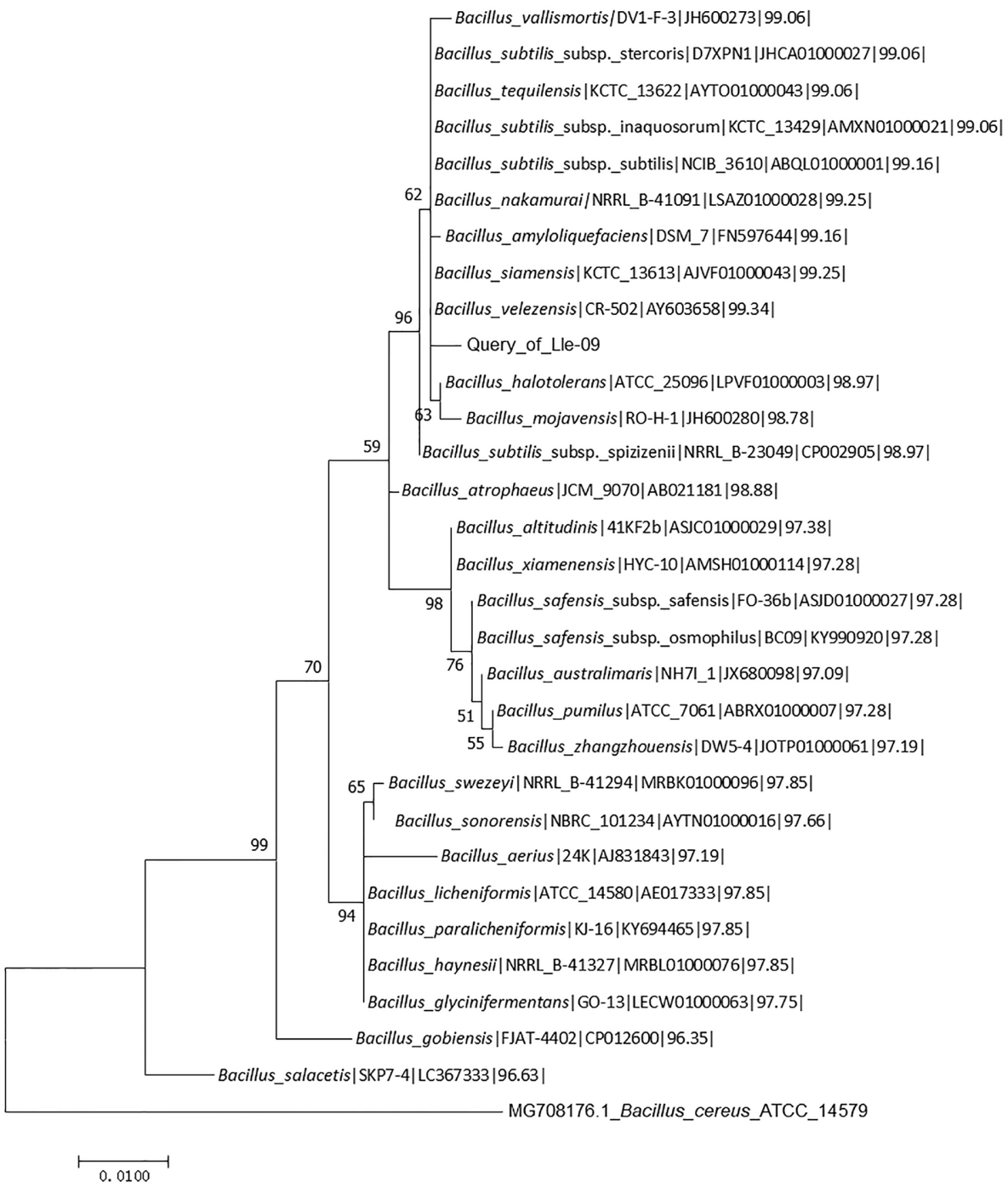

Fig. 2. Phylogenetic analysis of $16 \mathrm{~S}$ rRNA gene sequences of the bacterial endophyte Lle-9 isolated from Lilium leucanthum. Sequences were aligned through ClustalW using MEGA 7 software. Phylogenetic tree was constructed using Maximum Likelihood method. Bootstrap values are shown as percentages of 1000 replicates; values below $50 \%$ are not indicated. Bacillus cereus MG708176.1, ATCC14579 was used as an outgroup. 
A
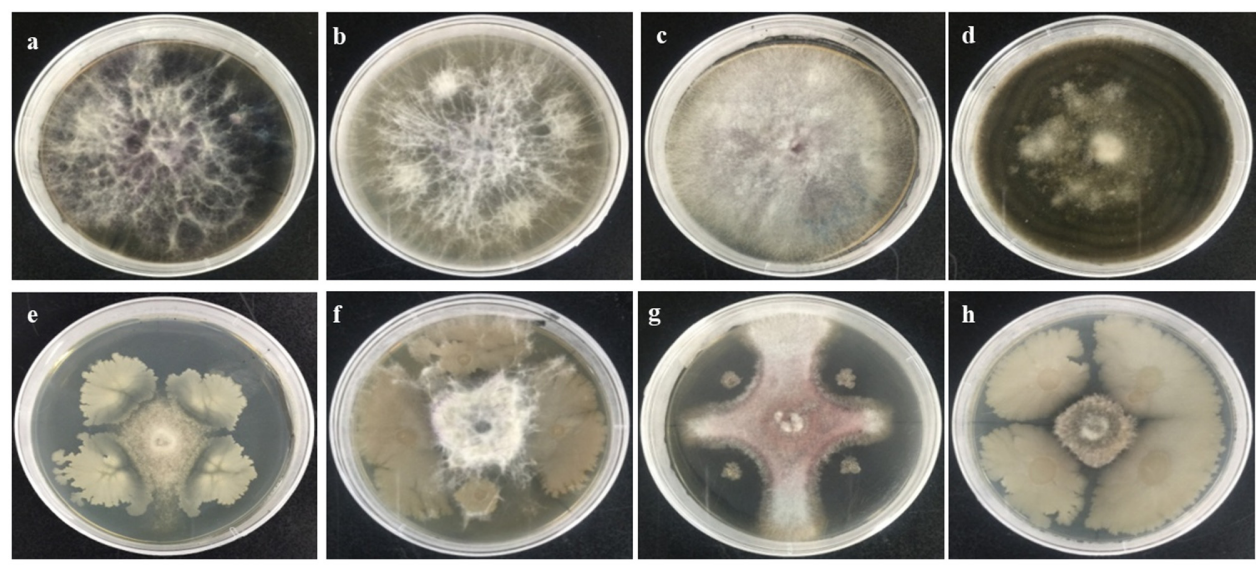

B

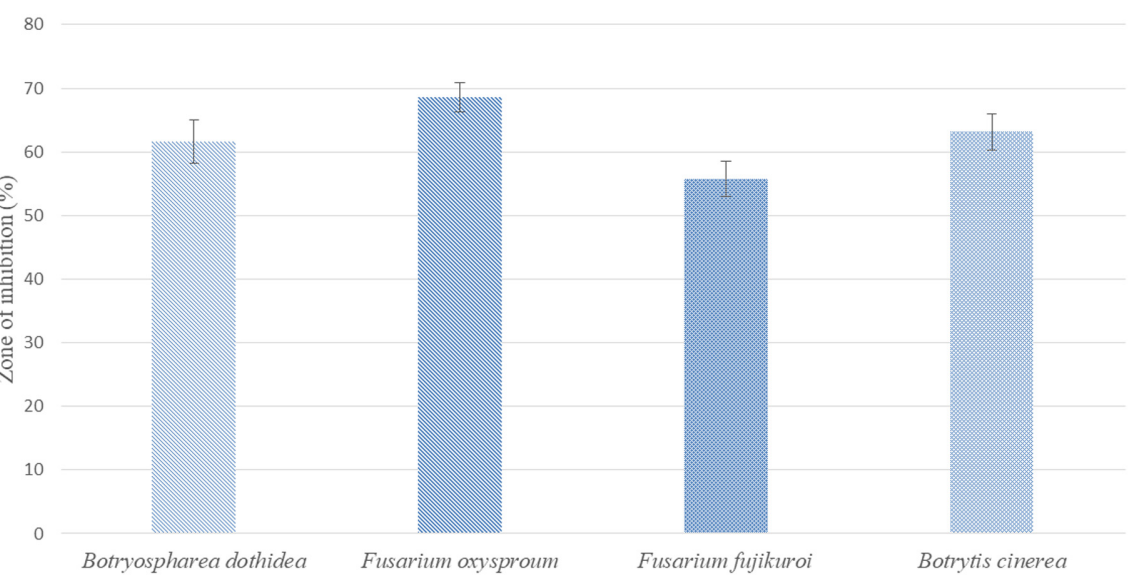

Fig. 3. In vitro antifungal activities of the isolated endophytic bacterial strain Lle-9 from Lilium leucanthum against four fungal pathogens. (A) $5 \mathrm{~mm}$ fungus plug was inoculated into the center of PDA medium surrounded by four spots of bacterial inoculum. Plates (A), (B), (C), and (D) are controls of Botryosphaeria dothidea, Fusarium oxysporum, Fusarium fujikuroi and Botrytis cinerea, respectively. Plates (E), (F), (G), and (H) contain dual cultures of Lle-9 and the fungal pathogens. (B) Antifungal activities were measured as size of the zones of inhibition of the pathogenic fungi. Zones of inhibition were expressed as percentages.

potential and disease-causing ability in bulbs of Asiatic lilium hybrid 'Tresor.' All pathogenic fungal strains revealed disease symptoms in bulbs of Tresor (Fig. S2). The strain Lle-9 exhibited considerable inhibition potential as revealed by zones of inhibition of the test pathogens on PDA plates. These high activities might be due to the release of some diffusible compound(s) against the test pathogens. Zones of inhibition of pathogenic fungi on PDA plates were measured as percentage values. The highest percentage of growth inhibition, i.e., $68.56 \pm$ $2.35 \%$, was observed against Fusarium oxysporum followed by $63.12 \pm 2.83 \%, 61.67 \pm 3.39 \%$, and $55.82 \pm 2.76 \%$ against Botrytis cinerea, Botryosphaeria dothidea, and Fusarium fujikuroi, respectively (Fig. 3B).

\section{Secondary Metabolite Analysis}

LC/MS was used to characterize the potential bioactive secondary compounds in ethyl acetate fraction. The compounds identified putatively in the ethyl acetate fraction of $B$. velezensis Lle- 9 are listed (Table S1). The compounds were characterized with molecular formula, $\mathrm{m} / \mathrm{z}$ measured, library m/z, GNPS score, and GNPS library ID. A total of 34 putative compounds were identified by using molecular networking workflow from the GNPS website. Raw LC-MS files were converted into mzXML using ProteoWizard 3.0.19140, and the mzXML file was uploaded to GNPS. The spectra in the network were then searched against GNPS' spectral libraries. The compounds identified in the ethyl acetate fraction of $B$. velezensis Lle- 9 were mostly diketopiperazines such as cyclo-dipeptides [cyclo(Ala-Leu), cyclo(D-Trp-L-Pro), cyclo-(Leu-Leu), and cyclo(Pro-Phe)], linear di-peptides [(Phe-Pro, Leu-Pro, Leu-His, Ile-Pro, Leu-Phe, Pro-Ile, Ile-Lys, His-Ile)], and tri-peptides [(Gln-Ile-Lys, and PyroGlu-Pro)]. In addition to these, some other bioactive compounds that were previously identified from various hosts as antifungal in nature were observed in the ethyl acetate fraction of Lle-9. The most important bioactive compounds were latrunculin A, 5alpha-hydroxy-6-ketocholesterol, (R)-S-lactoylglutathione, 1-(9ZOctadecenoyl)-sn-glycero-3-phosphoethanolamine, triamterene, (2E,6E,10Z)-12-hydroxy-10-(hydroxymethyl)-6methyl-2-(4-methylpent-3-enyl)dodeca-2,6,10-trienoic acid, 5S,12R,20-trihydroxy-6Z,8E,10E,14Z-eicosatetraenoic 
Table 1. Plant growth-promoting (PGP) traits of the endophytic strain Lle-9 of B. velezensis.

\begin{tabular}{|c|c|c|c|c|c|c|c|c|c|c|}
\hline \multirow[t]{2}{*}{$\mathrm{OA}$} & \multirow[t]{2}{*}{ ACC } & \multicolumn{4}{|c|}{$\begin{array}{l}\text { IAA }\left(\mu \mathrm{g} \mathrm{ml}^{-1}\right) \text { at different } \\
\text { tryptophan concentrations }\end{array}$} & \multicolumn{3}{|c|}{$\begin{array}{l}\text { Siderophores (psu) at different } \\
\text { Fe(III) citrate concentrations }\end{array}$} & \multirow[t]{2}{*}{ NA } & \multirow[t]{2}{*}{ PS } \\
\hline & & $0 \mathrm{mg} \mathrm{ml}^{-1}$ & $2 \mathrm{mg} \mathrm{ml}^{-1}$ & $4 \mathrm{mg} \mathrm{ml}^{-1}$ & $6 \mathrm{mg} \mathrm{ml}^{-1}$ & $0 \mu \mathrm{M}$ & $0.25 \mu \mathrm{M}$ & $3.0 \mu \mathrm{M}$ & & \\
\hline++ & ++ & $23.2 \pm 1.9$ & $79.7 \pm 3.3$ & $117.7 \pm 3.5$ & $165.7 \pm 5.8$ & $45.2 \pm 3.6$ & $35.1 \pm 1.3$ & $21.5 \pm 1.2$ & ++ & ++ \\
\hline
\end{tabular}

Results are means \pm standard deviation of three independent experiments with each treatment measured three times. Abbreviations are as follows: Organic acids (OA), ACC deaminase (ACC), Indole acetic acid (IAA), nitrogenase activity (NA), and phosphate solubilization (PS). Evaluation of the positivity to the tests; negative ( - ) shows absence of activity, while (+) shows lower to the highest activity $(+++)$.

acid, rubiadin, moxifloxacin, 9-hydroxy-5Z,7E,11Z,14Z-eicosatetraenoic acid, D-erythro-C18-sphingosine, citrinin, 2-arachidonoyllysophosphatidylcholine, and (2R)-5,8-dihydroxy-2-(2-hydroxyphenyl)-7-methoxy-2,3dihydrochromen-4-one.

\section{Plant Growth-Promoting (PGP) Assays}

The plant growth-promoting effects of Lle- 9 were assayed both qualitatively and quantitatively. According to our results, the endophytic bacterial strain Lle-9 showed positive results for all conducted assays.

\section{ACC (Deaminase) and Organic Acid Detection}

The ACC deaminase production was detected through a qualitative test based on change of color. The strain Lle-9 was found positive for the production of ACC deaminase (Table 1 and Fig. 4A).

The isolated strain Lle- 9 was assayed for production of organic acids through a qualitative test. Strain Lle- 9 showed moderate to high production of organic acids as revealed by the change of color from pink to yellow (Table 1 and Figs. 4A and 4B).

\section{Indole Acetic Acid}

Indole acetic acid (IAA) production in the isolated strain Lle-9 was detected through both qualitative and quantitative tests. Qualitative test confirmed IAA production in Lle-9 as revealed by the change of color of the culture supernatant from yellow to pink (Figs. 4A and 4C). Further, IAA was quantified in the strain at various tryptophan concentrations supplemented in the culture medium. The strain Lle-9 was able to produce IAA at different tryptophan concentrations (Table 1). Different tryptophan concentrations impacted the IAA production in the strain. The IAA content in the isolated strain increased with increasing tryptophan in the culture medium. The strain Lle-9 showed lower IAA content, i.e., $23.2 \pm 1.9 \mu \mathrm{g} \mathrm{ml}^{-1}$, at tryptophan concentration of $0 \mathrm{mg} \mathrm{ml}^{-1}$. However, the IAA content increased gradually with increasing the tryptophan concentrations from $0 \mathrm{mg} \mathrm{ml}^{-1}$ to

A

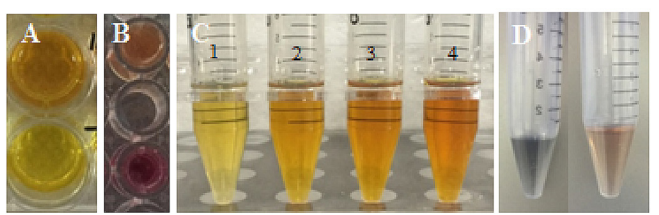

B

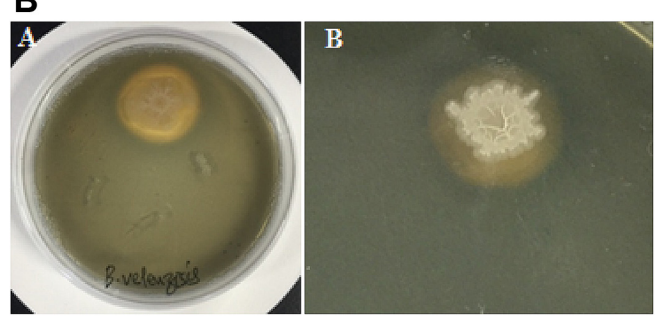

C

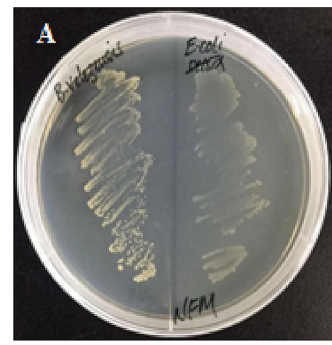

D

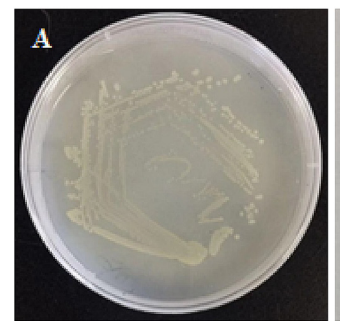

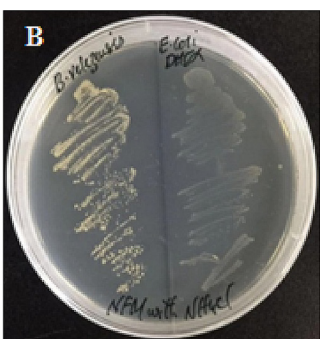

B

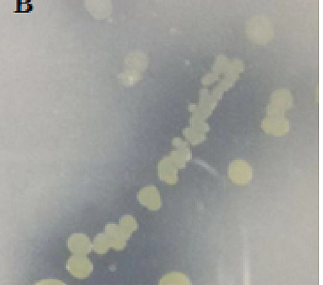

Fig. 4. Qualitative detection of plant growth-promoting traits in the isolated strain. (A) ACC deaminase activity of Lle-9 (A). Lower well was used as negative control. Organic acid production in the strain Lle-9 (B). Extreme lower well with pink color was used as negative control. IAA detection in the strain showing change of coloration from yellow to pink (C). The tubes numbered with $1,2,3$, and 4 show IAA detection at $0 \mathrm{mg} \mathrm{ml}^{-1}, 2 \mathrm{mg} \mathrm{ml}^{-1}, 4 \mathrm{mg} \mathrm{ml}^{-1}$, and $6 \mathrm{mg} \mathrm{ml}^{-1}$, respectively. Siderophore detection was confirmed by a change of color from blue to orange (D). (B) Siderophore detection on CAS blue agar plates. Siderophores were detected as a yellow/orange hallow surrounding the bacterial colonies (A). Closer look of the orange hallow surrounding the colony of Lle-9 (B). (C) Nitrogen-fixation by the endophytic strain Lle-9 of B. velezensis. The isolated strain was inoculated on nitrogen-deficient malate medium (NFM) and was assessed for growth in reference to non-nitrogenfixing E. coli DH5a. NFM medium (A); NFM supplemented with $5 \mathrm{mM} \mathrm{NH} 4 \mathrm{Cl}$ (B). (D) Phosphate solubilization assay of B. velezensis le-09. Growth of Lle-9 on NBRIP medium (A). Closer look of the clearing area surrounding bacterial colonies (B). 
$6 \mathrm{mg} \mathrm{ml}^{-1}$ in the culture medium. The endophytic strain Lle-9 produced $79.7 \pm 3.3 \mu \mathrm{g} \mathrm{ml}^{-1}, 117.7 \pm 3.5 \mu \mathrm{g} \mathrm{ml}^{-1}$, and $165.7 \pm 5.8 \mathrm{\mu g} \mathrm{ml}^{-1}$ at tryptophan concentrations of $2 \mathrm{mg} \mathrm{ml}^{-1}, 4 \mathrm{mg} \mathrm{ml}^{-1}$, and $6 \mathrm{mg} \mathrm{ml}^{-1}$ in the culture medium, respectively. These results suggest that the isolated strain Lle- 9 has the potential to produce high content of indole acetic acid even in the absence of exogenous tryptophan. Application of exogenous tryptophan had no negative impact on IAA production; rather, it increased the production and a positive correlation was observed between the IAA production and the increasing tryptophan concentrations.

\section{Siderophores}

Production of siderophores in Lle-9 was assayed both qualitatively and quantitatively at different $\mathrm{Fe}(\mathrm{III})$ citrate concentrations added to the culture medium. The endophytic strain Lle-9 was able to produce siderophores as confirmed through a change of color from blue to orange yellow (Figs. $4 \mathrm{~A}$ and $4 \mathrm{D}$ ). To further test whether an iron source has any impact on the production of siderophores, the strain was cultured in the liquid 284 medium supplemented with different Fe(III) citrate concentrations (Table 1). The strain Lle-9 showed high siderophore production when cultured in medium without Fe(III) citrate. The total siderophore quantity was reported as $51.3 \pm 3.8$ (psu) in the culture medium without addition of Fe(III) citrate. However, the siderophore accumulation by the strain declined as the quantities of Fe(III) citrate increased in the culture medium. Significantly greater decrease in siderophores was observed when $\mathrm{Fe}(\mathrm{III})$ citrate increased from $0 \mu \mathrm{M}$ to $0.25 \mu \mathrm{M}$. About $33.3 \pm 1.5$ (psu) siderophores were detected at $0.25 \mu \mathrm{M} \mathrm{Fe}$ (III) citrate in the medium. Further reduction in siderophores was observed when $\mathrm{Fe}(\mathrm{III})$ citrate concentration was increased to $3.0 \mu \mathrm{M}$. However, this was not significantly different from the quantities obtained at $0.25 \mu \mathrm{M}$. At $3.0 \mu \mathrm{M}$ Fe(III) citrate concentration, the strain Lle-9 accumulated $30.1 \pm 1.3$ (psu) siderophores. The siderophore production in the endophytic strain Lle- 9 was further assayed through a qualitative test using chrome azurol S (CAS) on agar plates. An orange/yellow hallow was observed around the colonies of Lle-9 indicating the production of siderophores. The strain Lle-9 was able to quench the iron from the dye complex that resulted in a change of color from blue to orange/yellow in the form of a hallow surrounding the bacterial colony (Fig. 4B). Further, the diameter of the yellow/orange hallow produced by the strain averaged $15.32 \pm 1.3 \mathrm{~mm}$. This test further confirmed the high potential of the isolated endophytic strain Lle-9 to produce siderophores.

\section{Potential for Nitrogen Fixation and Phosphate Solubilization}

The nitrogen-fixing potential of the isolated $B$. velezensis Lle- 9 was assessed by its ability to grow on nitrogenfree minimal medium (NFM). The Escherichia coli strain DH5a, which is unable to grow on nitrogen-free medium was used as a negative control. Both strains were cultured on nitrogen-free medium and medium supplemented with $5 \mathrm{mM} \mathrm{NH}_{4} \mathrm{Cl}$, which is a preferred source of nitrogen. Results revealed that the growth of $B$. velezensis Lle-9 was clearly visible on the NFM, while the Escherichia coli DH5a, which does not fix nitrogen, grew only on medium supplemented with reactive nitrogen (Fig. 4C). These results demonstrate the potential of the isolated endophytic $B$. velezensis Lle-9 to fix nitrogen.

The phosphate solubilization potential of the endophytic B. velezensis strain Lle-9 was assayed on solid NBRIP medium. In this medium, $\mathrm{Ca}_{3}\left(\mathrm{PO}_{4}\right)_{2}$ was provided as the sole source of inorganic phosphate. The endophytic strain Lle- 9 was able to grow on the medium for longer incubation time and it solubilized the inorganic phosphate as indicated by the clearing zone surrounding bacterial colonies (Fig. 4D).

\section{Plant Growth Promotion}

The plant growth-promoting potential of the isolated B. velezensis Lle-9 was further assessed on the vegetative growth and bulbs production of the Asiatic lilium hybrids 'Tresor' under greenhouse conditions. Bulbs of 'Tresor' were inoculated with the isolate strain before cultivation in soil pots. Upon completion of vegetative growth, a number of plant growth parameters like plant height, number of flowering shoots, leaf length, leaf width, stem diameter and weight of bulbs were measured between the inoculated and un-inoculated control plants. Our results revealed that inoculation of bulbs with $B$. velezensis Lle-9 resulted in significant increase in overall plant growth relative to control plants (Table 2). Inoculation of bulbs led to a significant increase in most of the tested growth parameters. The inoculated plants showed significantly high $(p \leq 0.05)$ increase in the number of flowering shoots, i.e. $3.47 \pm 0.30$, as compared to $2.73 \pm 0.45$ in un-inoculated control plants. Likewise, significant differences were observed in plant height between inoculated and un-inoculated plants (Fig. 5A, Table 2). Plants inoculated with Lle-9 showed plant height of $46.40 \pm 4.98 \mathrm{~cm}$ as compared to $41.21 \pm 4.69 \mathrm{~cm}$ of un-inoculated control plants. Leaf length also increased significantly in the inoculated plants relative to control plants. Inoculated plants showed significantly high ( $p \leq 0.05$ ) leaf length, i.e. $90.83 \pm 8.96 \mathrm{~mm}$, compared to $79.48 \pm 11.83$ $\mathrm{mm}$ in un-inoculated control plants. The inoculated plants showed improvement in leaf width relative to control plants; however, the differences were not significant. The B. velezensis Lle-9-inoculated plants showed $11.27 \pm 1.24$

Table 2. Growth improvement in the Asiatic hybrids 'Tresor' upon inoculation with isolated strain Lle-9.

\begin{tabular}{cccccccc}
\hline Treatments & $\begin{array}{c}\text { \# of flowering } \\
\text { shoots }\end{array}$ & $\begin{array}{c}\text { Plant height } \\
(\mathrm{cm})\end{array}$ & $\begin{array}{c}\text { Leaf length } \\
(\mathrm{mm})\end{array}$ & $\begin{array}{c}\text { Leaf width } \\
(\mathrm{mm})\end{array}$ & $\begin{array}{c}\text { Stem diameter } \\
(\mathrm{mm})\end{array}$ & $\begin{array}{c}\text { Bulb } \\
\text { weight }(\mathrm{g})\end{array}$ & $\begin{array}{c}\text { Root length } \\
(\mathrm{cm})\end{array}$ \\
\hline CK & $2.73 \pm 0.45$ & $41.21 \pm 4.69$ & $79.48 \pm 11.83$ & $10.10 \pm 1.24$ & $7.89 \pm 0.74$ & $12.66 \pm 1.20$ & $19.23 \pm 1.32$ \\
Lle-9 & $3.47 \pm 0.30^{*}$ & $46.40 \pm 4.98^{*}$ & $90.83 \pm 8.96^{*}$ & $11.27 \pm 1.24$ & $8.05 \pm 0.70$ & $14.45 \pm 0.91$ & $26.34 \pm 1.51^{*}$ \\
\hline
\end{tabular}

Values are averages $\pm \operatorname{SD}(n=15)$. Values with asterisk $\left(^{*}\right)$ represent significantly different based on Student's $t$-test $(p<0.05)$. 

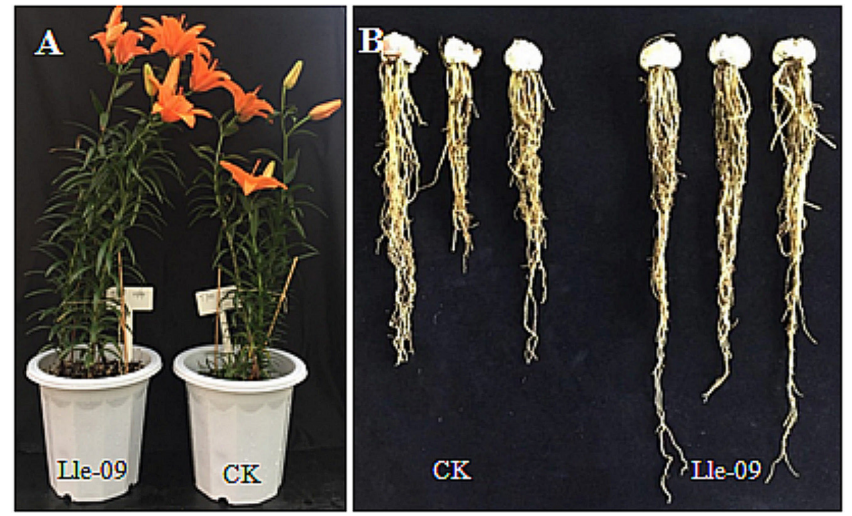

Fig. 5. Plant growth promotion in Tresor variety upon inoculation of bulbs with the isolated strain Lle-9. Phenotypic differences of Lle-9 inoculated and un-inoculated (control) plants (A). Differences in bulbs and root elongation between CK and Lle-9 inoculated plants (B).

$\mathrm{mm}$ average leaf width compared to $10.10 \pm 1.24 \mathrm{~mm}$ of the control plants. Similar increase was observed in stem diameter in the inoculated plants relative to control plants. However, the differences were not significantly different. Below ground characteristics of the plants such as size and weight of bulbs and root elongation were also found different between the inoculated and non-inoculated control plants. Bulb sizes and weights were slightly improved in the inoculated plants compared to control plants; however, the differences were not significant. Inoculated plants developed significantly longer roots $(26.34 \pm 1.51 \mathrm{~cm})$ as compared to non-inoculated control plants $(19.23 \pm 1.32 \mathrm{~cm})$ (Fig. $5 \mathrm{~B})$. Overall, these results revealed that the B. velezensis Lle- 9 was able to improve the vegetative and reproductive growth of lily plants upon inoculation.

\section{Discussion}

In the present study, a new endophytic bacterial strain Lle-9 of Bacillus velezensis was isolated from the bulbs of Lilium leucanthum. The isolated strain was assessed for its potential of antifungal activities against several fungal pathogens and it showed antimicrobial activity and resisted the growth and proliferation of these pathogens. The strain was identified to be B. velezensis through morphological and molecular analysis. Several plant growth promotion assays were conducted to confirm whether the strain could promote growth of the associated plants through several underlined mechanisms and whether it could also provide protection against disease-causing agents. Previous studies indicated that several B. velezensis strains isolated from various hosts, showed broadspectrum antimicrobial activities and plant growth promotion effects. Bacillus amyloliquefaciens FZB42, now recognized as a $B$. velezensis strain was reported in 2007 as the first gram-positive biocontrol bacteria to have its genome sequenced [36]. This isolate is now used as a model strain to promote plant growth and to confer disease resistance against broad-spectrum phytopathogens [37]. In addition, several other strains of $B$. velezensis have been used as antagonists of plant pathogens and as plant growth promoters in sustainable agriculture [12, 36, 38]. Further, a Bacillus velezensis strain CC09, isolated from healthy leaves of Cinnamomum camphora, showed immense potential as a new biocontrol agent, in control of many phytopathogenic diseases including wheat powdery mildew disease [39]. A B. velezensis strain NJAU-Z9, isolated from pepper rhizosphere, showed growth promotion effects in pepper [40].

In the present study, the isolated strain Lle-9 showed considerable antagonistic effects against fungal phytopathogens like Fusarium oxysporum, Botryosphaeria dothidea, Botrytis cinerea, and Fusarium fujikuroi. These pathogens have previously been reported to cause serious diseases in several crop plants [41]. These phytopathogens may cause diseases in Lilium species as revealed by a test confirming pathogenicity potential against cultivated species/varieties like Lilium davidii and Tresor. One of these phytopathogens, Fusarium fujikuroi, was isolated and identified from in vitro bulbs of Lilium wardii, a Lilium species. This could be a diseasecausing agent in Lilum wardii and other Lilum species. Interestingly, the isolated endophytic strain Lle-9 exhibited higher antifungal activities against all tested fungal pathogens and was found very effective against growth and proliferation of Fusarium fujikuroi.

The ability of the strain Lle-9 to suppress the growth of phytopathogens could be due to the presence of compounds and metabolites with antimicrobial properties. To provide evidence of this, the ethyl acetate fraction was assessed for potential secondary metabolites with bio-control properties. A number of secondary metabolites were identified and showed close homologies with the already known compounds with bio-control properties. Some of the prominent compounds and group of compounds, putatively identified from the isolated strain were diketopiperazines, cyclo-peptides, linear peptides, latrunculin A, 5alpha-hydroxy-6-ketocholesterol, (R)-Slactoylglutathione, triamterene, rubiadin, moxifloxacin, 9-hydroxy-5Z,7E,11Z,14Z-eicosatetraenoic acid, Derythro-C18-sphingosine, citrinin, and 2-arachidonoyllysophosphatidylcholine. Isolation of these secondary metabolites and compounds is evidence that the Lle- 9 has a high potential of restricting the growth and proliferation of disease-causing fungal pathogens. Our results are supported by previous identification of these bio-control compounds and metabolites from other Bacillus species. Diketopiperazines and linear di- and 
tripeptides were previously recovered from cultures of endophytic bacterial isolates. Syed-Ab-Rahman et al. [42] found diketopiperazines in the cultures of Bacillus amyloliquefaciens, Bacillus velezensis, and Acinetobacter sp. They further reported that the presence of these bioactive compounds resulted in resistance to phytophthora infection and plant growth promotion. A number of other studies also reported isolation of diketopiperazines (cyclo di-peptides) that resulted into broad-spectrum antimicrobial activities [43, 44]. Cyclo (Leu-Leu) found in Lactobacillus plantarum AF1 isolated from kimchi was found with antifungal activity against Aspergillus flavus [45]. Cyclo (Pro-Phe) in Bacillus amyloliquefaciens Q-426 was found to have a significant antifungal activity [46]. In addition, dipeptides and tripeptides have also been proved effective in conferring fungal resistance [47]. In the present study, latrunculin A was detected in the Lle-9 extract. This compound was previously shown as having fungistatic, fungicidal and fungilytic effects on the budding yeast Saccharomyces cerevisiae [48]. Triamterene, found in the ethyl acetate fraction of Lle- 9 was previously found in ayurvedic medicine, Salmali niryasa, from a medicinal plant, Bombax ceiba [49]. The salmali resin had shown strong antimicrobial and antioxidant activities. Rubiadin, a bioactive compound detected in the present study was isolated from the roots of Morinda elliptica $\mathrm{L}$. (Rubiaceae) [50]. This compound demonstrated anti-HIV, cytotoxic and antimicrobial activities [51]. Recently, rubiadin (AQ1) and rubiadin 1-methyl ether (AQ2), two photosensitizing anthraquinones (AQs) isolated from Heterophyllaea pustulata, showed reduction in biofilms formation of Candida tropicalis, a common cause of fungal infections [52]. Moxifloxacin was another important bioactive compound detected in the present study. This compound was previously shown as having antimicrobial effects [53]. The presence of these bioactive compounds might be responsible for the significant antifungal activities of Lle-9 against the tested fungal pathogens.

Previous studies have revealed that the plant growth-promoting traits in isolated strains of Bacillus species were correlated with several mechanisms such as production of organic acids, siderophores, lowering plant ethylene levels by ACC deaminase production, synthesis of plant growth-regulating hormones like indole-acetic acid and cytokinins, nitrogen fixation and phosphate solubilization [54]. In the present study, we confirmed through various tests that the isolated strain Lle-9 possessed plant growth-promoting traits. The isolated strain Lle-9 was capable of producing organic acids as revealed by a qualitative test. Organic acids play an important role in plant growth promotion and defense against phytopathogens [55].

Moreover, production of 1-aminocyclopropane-1-carboxylate (ACC) deaminase is one of the important characteristics of plant growth-promoting microbes and endophytes. ACC deaminase cleaves ACC, the immediate precursor of the plant hormone ethylene, to produce $\alpha$-ketobutyrate and ammonia [56]. Ethylene serves as an important signaling molecule in plants under biotic and abiotic stresses and results in plant growth inhibition [57]. Previous studies have reported that inoculation of plants with ACC deaminase-producing microbes decreased ethylene levels that resulted in decreased inhibition of plant growth under biotic and abiotic stresses $[3,58]$. Previous studies showed that improvement of several crops, inoculated with Bacillus species, might partly be due to the production of ACC deaminase. The $B$. velezensis strain $\mathrm{BACO}_{3}$ was reported to produce ACC deaminase, indole acetic acid, and ammonia, resulting in improved plant growth in terms of high biomass of leaves and roots in radish [59]. Bacillus species isolated from seeds of commercial tomato varieties exhibited multiple plant growth-promoting traits such as production of ACC deaminase, IAA, siderophores, and potential of nitrogen fixation and phosphate solubilization [60]. One of the isolates, HYT-12-1, identified as Bacillus subtilis, showed the highest ACC deaminase activity, which resulted in growth enhancement of tomato seedlings under greenhouse conditions. Consistent with the mentioned reports, the isolated strain Lle-9 in the present study exhibited ACC deaminase activity that might partly contributed to the improvement of growth of lily varieties upon inoculation.

Indole acitic acid is one of the important auxins that directly support plant growth and productivity. The ability of PGPRs including Bacillus species is partly attributed to their potential of IAA production that directly promotes growth of associated plants. In this connection, the role of the tryptophan precursor of IAA is important as considerable amounts of IAA are produced in the presence of excess tryptophan [61]. In the present study, the exogenous tryptophan concentrations had no negative impact on IAA production in Lle-9 and a positive correlation was observed between the tryptophan concentrations and IAA production. It seems the isolated strain Lle-9 produced IAA in a tryptophan-dependent pathway. Our results are supported by previous reports where application of exogenous tryptophan enhanced the IAA production. The Bacillus amyloliquefaciens strain FZB42, now a $B$. velezensis strain, was reported with production of IAA and its capacity increased five-fold with application of exogenous tryptophan [62]. Bacillus thuringiensis and Bacillus cereus isolated from soil samples exhibited IAA production in the absence and presence of L-tryptophan [63]. It was reported that the IAA production in the isolates increased with increasing concentrations of exogenous tryptophan.

Iron is an essential element necessary for growth of plants and microorganisms. However, it is abundantly present in soil in the form of insoluble $\mathrm{Fe}^{3+}$ oxy-hydroxides. Plant-associated microbes reduce $\mathrm{Fe}^{3+}$ to $\mathrm{Fe}^{2+}$ with the help of ferrireductases or solubilize it with extracellular $\mathrm{Fe}^{3+}$ chelators called 'siderophores' [64]. These soluble $\mathrm{Fe}^{3+}$-siderophore complexes are then available to both plants and microbes. Species of the genus Bacillus were previously reported with production of siderophores. Chen et al. (2007) reported siderophore bacillibactin production in B. amyloliquefaciens FZB42, now B. velezensis, and described that the siderophore synthesis in the strain was catalyzed by nonribosomal peptide synthetases. They further reported that production of high concentrations of siderophore bacillibactin in the FZB42 strain inhibited the growth of phytopathogenic bacterial and fungal competitors by depriving them of essential iron ions. Some recent studies also reported production of siderophores in Bacillus species $[65,66]$. Kesaulya et al. [65] reported siderophore production in the isolated Bacillus sp. from potato rhizospheric soil. They further reported that the isolated strain inhibited the pathogen 
causing banana wilt disease. These reports reveal that siderophore production by the plant-associated microbes not only helps plants acquire access to limited iron supply in the soil but also confers plants with a selective advantage over the pathogenic microbes by depriving them of essential iron that leads to disease resistance. In the present study, the high siderophore production in the isolated strain Lle-9 might partly be responsible for considerable antagonistic effects against the tested pathogenic strains. Quantitative evaluation of siderophores was conducted under different iron concentrations. Maximum siderophore production was observed in the absence of $\mathrm{Fe}$ (III) citrate in the medium. However, increasing iron concentrations in the medium resulted in reduction in siderophore accumulation. These results are supported by previous reports where an inverse relationship was observed between siderophore production and different iron concentrations in the medium [67, 68].

Nitrogen is an essential and vital element for the normal growth and developments of plants. Many of the isolated PGPRs including species of Bacillus were found with the ability to fix atmospheric nitrogen. Syed-AbRahman et al. [42] reported isolation of bacterial species including B. amyloliquefaciens (UQ154) and B. velezensis (UQ156) from soils of Arabidopsis plants. The Bacillus species were found with nitrogen-fixing ability. Other Bacillus species, which were isolated from Sophora Alopecuroides root nodules and rice showed nitrogen-fixing potential $[69,70]$. Likewise, in the present study, the isolated endophytic B. velezensis strain Lle-9 showed nitrogen fixation ability by growth on nitrogen-free medium. In addition, the strain was able to solubilize inorganic phosphate on a solid NBRIP medium supplemented with $\mathrm{Ca}_{3}\left(\mathrm{PO}_{4}\right)_{2}$ as the sole source of phosphate. Phosphate solubilization is one of the most important plant growth-promoting traits associated with endophytic bacteria.

The different strains belonging to the genus Bacillus have been proved very effective as biocontrol agents and biofertilizers in sustainable agriculture [38,71]. Utilization of these bacteria has been found effective in controlling pathogenic microbes, exerting beneficial effects on plant growth and facilitating nutrient accessibility and assimilation [72-74]. Having these beneficial effects, the isolated strain Lle-9 was assessed for growthpromoting effects on the Asiatic lilium hybrid 'Tresor' under greenhouse conditions. Inoculated plants exhibited growth improvement and significant increases were observed for several growth parameters between the inoculated and non-inoculated control plants. Inoculated plants not only showed improved plant growth and bulb production but also produced a high number of flowers per plant. The improved growth performance in the Asiatic lilium hybrids 'Tresor' upon inoculation of Lle-9 might be due to the growth-promoting effects as were evaluated through several qualitative and quantitative tests. Previous studies also reported similar growth improvement when plants were inoculated with the isolated strains of Bacillus velezensis $[75,40]$. The isolated $B$. velezensis strain Lle-9 showed antagonistic effects against the broad-spectrum fungal pathogens. These enhanced antifungal activities might be due to the presence of a number of bioactive compounds, which have previously been recognized as antimicrobial in nature. Moreover, the strain Lle- 9 exhibited several plant growth-promoting traits, which were reflected in the improved vegetative and reproductive growth of lily plants upon inoculation. Owing to these beneficial antifungal and plant growth-promoting properties, the B. velezensis strain Lle- 9 may be a good choice to be utilized as a source of bio-fertilizer and bio-control agent in sustainable agriculture.

\section{Acknowledgment}

This research was funded by the National Key Research and Development Program of China [2017YFD0501005]; Science and Technology Innovation Ability Construction of Beijing Academy of Agricultural and Forestry Sciences (KJCX20170415).

\section{Conflict of Interest}

The authors have no financial conflicts of interest to declare.

\section{References}

1. Kloepper JW, Leong J, Teintze M, Schroth MN. 1980. Enhancing plant growth by siderophores produced by plant growth-promoting rhizobacteria. Nature 286: 885-886.

2. Rodriguez H, Fraga R. 1999. Phosphate solubilizing bacteria and their role in plant growth promotion. Biotechnol. Adv. 17:319-339.

3. Glick BR, Cheng Z, Czarny J, Duan J. 2007. Promotion of plant growth by ACC deaminase-producing soil bacteria. Eur. J. Plant Pathol. 119: 329-339.

4. Velkov T, Thompson PE, Nation RL, Li J. 2010. Structure - activity relationships of polymyxin antibiotics. J. Med. Chem. 53: 18981916.

5. Kim YC, Leveau J, McSpadden Gardener BB, Pierson EA, Pierson LS, et al. 2011. The multifactorial basis for plant health promotion by plant-associated bacteria. Appl. Environ. Microbiol. 77: 1548-1555.

6. Tendulkar SR, Saikumari YK, Patel V, Raghotama S, Munshi TK, Balaram P, et al. 2007. Isolation, purification and characterization of an antifungal molecule produced by Bacillus licheniformis BC98, and its effect on phytopathogen Magnaporthe grisea. Appl. Microbiol. 103: 2331-2339.

7. Wang J, Liu J, Chen H, Yao J. 2007. Characterization of Fusarium graminearum inhibitory lipopeptide from Bacillus subtilis IB. Appl. Microbiol. Biotechnol. 76: 889-894.

8. Romero D, Perez-Garcia A, Rivera ME, Cazorla FM, de Vicente A. 2004. Isolation and evaluation of antagonistic bacteria towards the cucurbit powdery mildew fungus Podosphaera fusca. Appl. Microbiol. Biotechnol. 64: 263-269.

9. Borriss R, Chen XH, Rueckert C, Blom J, Becker A, Baumgarth B, et al. 2011. Relationship of Bacillus amyloliquefaciens clades associated with strains DSM7 ${ }^{\mathrm{T}}$ and FZB42 $2^{\mathrm{T}}$ : a proposal for Bacillus amyloliquefaciens subsp. amyloliquefaciens subsp. nov. and Bacillus amyloliquefaciens subsp. plantarum subsp. nov. based on complete genome sequence comparisons. Int. J. Syst. Evol. Microbiol. 61: 1786-1801. 
10. Perez-Garcia A, Romero D, de Vicente A. 2011. Plant protection and growth stimulation by microorganisms: biotechnological applications of Bacilli in agriculture. Curr. Opin. Biotechnol. 22: 187-193.

11. Wang LT, Lee FL, Tai CJ, Kuo HP. 2008. Bacillus velezensis is a later heterotypic synonym of Bacillus amyloliquefaciens. Int. J. Syst. Evol. Microbiol. 58: 671-675.

12. Dunlap CA, Kim SJ, Kwon SW, Rooney AP. 2015. Phylogenomic analysis shows that Bacillus amyloliquefaciens subsp. plantarum is a later heterotypic synonym of Bacillus methylotrophicus. Int. J. Syst. Evol. Microbiol. 65: 2104-2109.

13. Madhaiyan M, Poonguzhali S, Kwon SW, Sa TM. 2010. Bacillus methylotrophicus sp. nov, a methanol-utilizing, plant-growthpromoting bacterium isolated from rice rhizosphere soil. Int. J. Syst. Evol. Microbiol. 60: 2490-2495.

14. Chowdhury SP, Dietel K, Rändler M, Schmid M, Junge H, Borriss R. et al. 2013. Effects of Bacillus amyloliquefaciens FZB42 on lettuce growth and health under pathogen pressure and its impact on the rhizosphere bacterial community. PLoS One 8: e68818.

15. Chowdhury SP, Hartmann A, Gao X, Borriss R. 2015. Biocontrol mechanism by root-associated Bacillus amyloliquefaciens FZB42a review. Front. Microbiol. 6: 780 .

16. Rong LP, Lei JJ, Wang C. 2011. Collection and evaluation of the genus Lilium resources in Northeast China. Genet. Resour. Crop Evol. 58: 115-123.

17. Chau CF, Wu SH. 2006. The development of regulations of Chinese herbal medicines for both medicinal and food uses. Trends Food Sci. Technol. 17: 313-323.

18. You X, Xie C, Liu K, Gu Z. 2010. Isolation of non-starch polysaccharides from bulb of tiger lily (Lilium lancifolium Thunb) with fermentation of Saccharomyces cerevisiae. Carbohydr. Polym. 81: 35-40.

19. Schulz B, Boyle C, Draeger S, Rommert AK, Krohn K. 2002. Endophytic fungi: a source of novel biologically active secondary metabolites. Mycol. Res. 106: 996-1004.

20. Tan RX, Zou WX. 2001. Endophytes: a rich source of functional metabolites. Nat. Prod. Rep. 18: 448-459.

21. Strobel GA, Sears J, Kramer R, Sidhu RS, Hess WM. 1996. Taxol from Pestalotiopsis microspora an endophytic fungus of Taxus wallachiana. Microbiology 142: 435-440.

22. Vincent JM, Humphrey B. 1970. Taxonomically significant group antigens in Rhizobium. J. Gen. Microbiol. 63: 379-382.

23. Tamura K, Peterson D, Peterson N, Stecher G, Nei M, Kumar S. 2011. MEGA5: molecular evolutionary genetics analysis using maximum likelihood, evolutionary distance, and maximum parsimony methods. Mol. Biol. Evol. 28: 2731-2739.

24. Khamna S, Yokota A, Lumyong S. 2009. Actinomycetes isolated from medicinal plant rhizospheric soils: diversity and screening of antifungal compounds, indole-3-acetic acid and siderophore production. World J. Microbiol. Biotechnol. 25: 649-655.

25. Lee S, Oh DG, Lee S, Kim G, Lee J, Son Y, et al. 2015. Chemotaxonomic metabolite profiling of 62 indigenous plant species and its correlation with bioactivities. Molecules 20: 19719-19734.

26. Wang M, Carver JJ, Phelan VV, Sanchez LM, Garg N, Peng Y, et al. 2016. Sharing and community curation of mass spectrometry data with global natural products social molecular networking. Nat. Biotechnol. 34: 828-837.

27. Chambers MC, Maclean B, Burke R, Amodei D, Ruderman DL, Neumann S, et al. 2012. A cross-platform toolkit for mass spectrometry and proteomics. Nat. Biotechnol. 30: $918-920$.

28. Belimov AA, Hontzeas N, Safronova VI, Demchinskaya SV, Piluzza G, Bullitta S, et al. 2005. Cadmium-tolerant plant growthpromoting bacteria associated with the roots of Indian mustard (Brassica juncea L. Czern.). Soil. Biol. Biochem. 37: 241-250.

29. Truyens S, Jambon I, Croes S, Janssen J, Weyens N, Mench M, et al. 2014. The effect of long-term cd and ni exposure on seed endophytes of Agrostis capillaris and their potential application in phytoremediation of metal-contaminated soils. Int. J. Phytorem. 16: 643-659.

30. Cunningham JE, Kuiack C. 1992. Production of citric and oxalic acids and solubilization of calcium-phosphate by Penicillium bilaii. Appl. Environ. Microbiol. 58: 1451-1458.

31. Gordon SA, Weber RP. 1951. Colorimetric estimation of indoleacetic acid. Plant. Physiol. 26: 192-195.

32. Schwyn B, Neilands JB. 1987. Universal chemical assay for the detection and determination of siderophores. Anal. Biochem. 160: 4756.

33. Doebereiner J. 1994. Isolation and identification of aerobic nitrogen fixing bacteria. In: Alef K, Nannipieri P, pp. 134-141 (eds.), Methods in Applied Soil Microbiology and Biochemistry. Cambridge, MA, USA, Academic.

34. Bashan Y, Holguin G, Lifshitz R. 1993. Isolation and characterization of plant growth-promoting rhizobacteria. In: Glick BR, Thompson JE, pp. 331-345 (eds.), Methods in Plant Molecular Biology and Biotechnology. BocaRaton, FL, USA, CRC Press.

35. Mehta S, Nautiyal CS. 2001. An efficient method for qualitative screening of phosphate-solubilizing bacteria. Curr. Microbiol. 43: 5156.

36. Chen XH, Koumoutsi A, Scholz R, Eisenreich A, Schneider K, Heinemeyer I, et al. 2007. Comparative analysis of the complete genome sequence of the plant growth-promoting bacterium Bacillus amyloliquefaciens FZB42. Nat. Biotechnol. 25: 1007-1014.

37. Rabbee MF, Ali MD, Choi J, Hwang BS, Jeong SC, Baek KH. 2019. Bacillus velezensis: A valuable member of bioactive molecules within plant microbiomes. Molecules 24: 1046.

38. Yao A, Dr HB, Karimov S, Boturov U, Sanginboy S, Sharipov AK. 2006. Effect of FZB $24^{\star}$ Bacillus subtilis as a biofertilizer on cotton yields in field tests. Arch. Phytopathol. Plant. Protect. 39: 323-328.

39. Cai XC, Liua CH, Wang BT, Xuea YR. 2016. Genomic and metabolic traits endow Bacillus velezensis CC09 with a potential biocontrol agent in control of wheat powdery mildew disease. Microbiol. Res. 196: 89-94.

40. Zhang Y, Gao X, Wang S, Zhu C, Li R, Shen Q. 2018. Application of Bacillus velezensis NJAU-Z9 enhanced plant growth associated with efficient rhizospheric colonization monitored by qpcr with primers designed from the whole genome sequence. Curr. Microbiol. 75: 1574-1583.

41. Horst RK. 2013. Field manual of diseases on fruits and vegetables. Springer Science+Business Media Dordrecht.

42. Syed-Ab-Rahman SF, Carvalhais LC, Chua E, Xiao Y, Wass TJ, Schenk PM. 2018. Identification of soil bacterial isolates suppressing different Phytophthora spp. and promoting plant growth. Front. Plant. Sci. 9: 1502.

43. Martínez-Luis S, Ballesteros J, Gutiérrez M. 2011. Antibacterial constituents from the octocoral-associated bacterium Pseudoalteromonas sp. Revista Latinoamericana Química. 39: 75-83.

44. Nishanth Kumar S, Mohandas C, Siji J, Rajasekharan K, Nambisan B. 2012. Identification of antimicrobial compound, diketopiperazines, from a Bacillus sp. N strain associated with a rhabditid entomopathogenic nematode against major plant pathogenic fungi. J. Appl. Microbiol. 113: 914-924.

45. Yang E, Chang H. 2010. Purification of a new antifungal compound produced by Lactobacillus plantarum AF1 isolated from kimchi. Int. J. Food. Microbiol. 139: 56-63.

46. Wang XM, Bai YJ, Cai YJ, Zheng XH. 2017. Biochemical characteristics of three feruloyl esterases with a broad substrate spectrum from Bacillus amyloliquefaciens H47. Process. Biochem. 53: 109-115.

47. Gill K, Kumar S, Xess I, Dey S. 2015. Novel synthetic anti-fungal tripeptide effective against Candida krusei. Ind. J. Med. Microbiol. 33: $110-116$.

48. Kloepper JW, Leong J, Teintze M, Schroth MN. 1980. Enhancing plant growth by siderophores produced by plant growth-promoting rhizobacteria. Nature 286: 885-886. 
49. Rao MRK, Philip S, Kumar MH, Saranya Y, Divya D, Prabhu K. 2015. GC-MS analysis, antimicrobial, antioxidant activity of an Ayurvedic medicine, Salmali Niryasa. J. Chem. Pharma. Res. 7: 131-139.

50. Ismail NH, Ali AM, Aimi N, Kitajima M, Takayama H, Lajis NH. 1997. Anthraquinones from Morinda elliptica. Phytochemistry 45: $1723-1725$.

51. Ali AM, Ismail NH, Mackeen MM, Yazan LS, Mohamed SM, Ho ASH, et al. 2000. Antiviral, cyototoxic and antimicrobial activities of anthraquinones isolated from the roots of Morinda elliptica. Pharma. Biol. 38: 298-301.

52. Marioni J, da Silva MA, Cabreraa JL, Núñez Montoyaa SC, Paraje MG. 2016. The anthraquinones rubiadin and its 1-methyl ether isolated from Heterophyllaea pustulata reduces Candida tropicalis biofilms formation. Phytomedicine 23: 1321-1328.

53. Matoba AY. 2012. Fungal keratitis responsive to Moxifloxacin monotherapy. Cornea 31: 1206-1209.

54. Lopes R, Tsui S, Gonçalves PJRO, de Queirozm MV. 2018. A look into a multifunctional toolbox: endophytic Bacillus species provide broad and underexploited benefits for plants. World. J. Microbiol. Biotechnol. 34: 94

55. de Werra P, Péchy-Tarr M, Keel C, Maurhofer M. 2009. Role of gluconic acid production in the regulation of biocontrol traits of Pseudomonas fluorescens CHA0. Appl. Environ. Microbiol. 75: 4162-4174.

56. Todorovic B, Glick BR. 2008. The interconversion of ACC deaminase and D-cysteine desulfhydrase by directed mutagenesis. Planta 229: 193-205.

57. Abeles FB, Morgan PW, Saltveit Jr ME. 1992. Ethylene in plant biology, pp. 1-13. 2nd edn. San Diego, Academic Press.

58. Farwell AJ, Vesely S, Nero V, Rodriguez H, McCormack K, Shah S, et al. 2007. Tolerance of transgenic canola plants (Brassica napus) amended with plant growth-promoting bacteria to flooding stress at a metal-contaminated field site. Environ. Pollut. 147: $540-545$.

59. Meng Q, Jiang H, Hao JJ. 2016. Effects of Bacillus velezensis strain BAC03 in promoting plant growth. Biol. Cont. 98: 18-26.

60. Xu M, Sheng J, Chen L, Men Y, Gan L, Guo S, et al. 2014. Bacterial community compositions of tomato (Lycopersicum esculentum Mill.) seeds and plant growth promoting activity of ACC deaminase producing Bacillus subtilis (HYT-12-1) on tomato seedlings. World. J. Microbiol. Biotechnol. 30: 835-845.

61. Patten CL, Blakney AJC, Coulson TJD. 2013. Activity, distribution and function of indole-3-acetic acid biosynthetic pathways in bacteria. Crit. Rev. Microbiol. 39: 395-415.

62. Idris EE, Iglesias DJ, Talon M, Borriss R. 2007. Tryptophan-dependent production of indole-3-acetic acid (IAA) affects level of plant growth promotion by Bacillus amyloliquefaciens FZB42. Mol. Plant. Microbe. Interact. 20: 619-626

63. Chagas Junior Af, De Oliveira AG, De Oliveira LA, Dos Santos Gr, Chagas LFB, Lopes da Silva AL, Luz Costa J. 2015. Production of indole-3-acetic acid by bacillus isolated from different soils. Bulg. J. Agric. Sci. 21:282-287.

64. Raza W, Shen Q. 2010. Growth, Fe3 + reductase activity, and siderophore production by Paenibacillus polymyxa SQR-21 under differential iron conditions. Curr. Microbiol. 61:390-5.

65. Kesaulya H, Hasinu JV, Tuhumury GNC. 2018. Potential of Bacillus spp produces siderophores in suppressing the wilt disease of banana plants. IOP Conference Series: Earth Environ. Sci. 102(1): 012016.

66. Ferreira CMH, Vilas-Boas A, Sousa CA, Soares HMVM, Soares EV. 2019. Comparison of five bacterial strains producing siderophores with ability to chelate iron under alkaline conditions. AMB Express 9: 78.

67. Tailor AJ, Joshi BH. 2012. Characterization and optimization of siderophore production from Pseudomonas fluorescens strain isolated from sugarcane rhizosphere. J. Environ. Res. Dev. 6: 688-694.

68. Kumar VS, Menon S, Agarwal H, Gopalakrishnan D. 2017. Characterization and optimization of bacterium isolated from soil samples for the production of siderophores. Resource-Efficient Technol. 3: 434-439.

69. Zhao L, Xu Y, Sun R, Deng Z, Yang W, Wei G. 2011. Identification and characterization of the endophytic plant growth prompter Bacillus cereus strain mq23 isolated from Sophora alopecuroides root nodules. Braz. J. Microbiol. 42: 567-575.

70. Shen FT, Yen JH, Liao CS, Chen WC, Chao YT. 2019. Screening of rice endophytic biofertilizers with fungicide tolerance and plant growth-promoting characteristics. Sustainability 11: 1133.

71. Borriss R. 2011. "Use of plant-associated Bacillus strains as biofertilizers and biocontrol agents," in Bacteria in Agrobiology. In: Maheshwari DK, pp. 41-76 (ed.), Plant Growth Responses. Heidelberg, Springer.

72. Compant S, Brader G, Muzammil S, Sessitsch A, Lebrihi A, Mathieu F. 2013. Use of beneficial bacteria and their secondary metabolites to control grapevine pathogen diseases. BioControl 58: 435-455.

73. Bach E, Dos Santos Seger GD, De Carvalho Fernandes G, Lisboa BB, Passaglia LMP. 2016. Evaluation of biological control and rhizosphere competence of plant growth promoting bacteria. Appl. Soil. Ecol. 99: 141-149.

74. van Lenteren JC, Bolckmans K, Köhl J, Ravensberg WJ, Urbaneja A. 2018. Biological control using invertebrates and microorganisms: plenty of new opportunities. BioControl 63: 39-59.

75. Fan B, Wang C, Song X, Ding X, Wu L, Wu H, et al. 2018. Bacillus velezensis FZB42 in 2018: the gram-positive model strain for plant growth promotion and biocontrol. Front. Microbiol. 9: 2491. 